\title{
3D Target Localization by Using Particle Filter with Passive Radar Having one Non-cooperative Transmitter and one Receiver
}

\author{
Anas Mahmoud Almanofi, Adnan Malki, and Ali Kazem
}

\begin{abstract}
In a passive radar system, localizing a target in Cartesian space is achieved by using one of the following bistatic geometries: multiple non-cooperative transmitters with one receiver, one non-cooperative transmitter with multiple receivers, or one non-cooperative transmitter with one receiver. In this paper, we propose a new method for localizing a target in Cartesian space by passive radar having the bistatic geometry "one non-cooperative transmitter and one receiver". This method depends on using two consecutive particle filters for estimating and analyzing the Doppler frequency and time delay of the target's echo signal. The theoretical analysis of the proposed method is presented, and its efficiency is verified by simulating the passive radar system with a Digital Video BroadcastingTerrestrial (DVB-T) transmitter.
\end{abstract}

Index Terms - Passive Radar, Target Localization, Estimation of Target Coordinates, Non-cooperative Transmitter, Receiver, Particle Filter, Doppler Frequency, Time Delay.

\section{INTRODUCTION}

$\mathrm{P}$ ASSIVE radar is a special bistatic radar that does not have dedicated transmitters, whereas it detects and tracks targets by processing electromagnetic reflections corresponding to non-cooperative transmitters [1]. The common structure of its receiver consists of the following two receiving channels: First, the surveillance channel for receiving targets' echoes and multipath signals. Second, the reference channel for receiving the reference signal (direct signal), which is used for detecting targets' echoes signals [2, 3]. It has many advantages compared to active radar, such as lower cost and better immunity to jamming [3, 4].

Many researches have been conducted studying this radar, such as studying of signals of non-cooperative transmitters (e.g. Frequency Modulation (FM) radio, Global System for Mobile communication (GSM), Digital Video BroadcastingTerrestrial (DVB-T), and Digital Audio Broadcasting (DAB))

Manuscript received July 9, 2020; revised December 5, 2020. Date of publication March 19, 2021. Date of current version March 19, 2021. The associate editor prof. Zoran Blažević has been coordinating the review of this manuscript and approved it for publication.

Authors are with the Higher Institute for Applied Sciences and Technology (HIAST), Damascus, Syria (e-mails: \{anas.almanofi, adnan.malki, ali.kazemn@hiast.edu.sy).

Digital Object Identifier (DOI): 10.24138/jcomss.v17i1.1110
$[1,4,5]$, suppression of the interference affecting the surveillance channel $[6,7]$, improving the detection of targets' echoes signals $[6,8,9]$, and estimation of targets' parameters (e.g. velocity and coordinates) [10]-[16].

Passive radar estimates target's Coordinates (or localizes a target in Cartesian space) by using one of the following two methods: First, estimating and processing the bistatic time delay corresponding to the transmitter-receiver pairs in the bistatic geometries "multiple non-cooperative transmitters or multiple receivers" [11]-[14]. Second, estimating and analyzing parameters of the target's echo signal in the bistatic geometry "one non-cooperative transmitter and one receiver" [15]. The first method has the following disadvantages compared to the second method: a ghost target phenomenon and extra signal processing $[1,15]$.

In this paper, we propose a new method for estimating target's coordinates by passive radar that has only one noncooperative transmitter and one receiver. This method depends on estimating and analyzing the Doppler frequency and time delay of the target's echo signal. We suppose that the velocity of the studied target changes in a non-linear way, so we should choose one of the non-linear tracking filters for estimating the two mentioned parameters. There are different types for these filters, such as Extended Kalman Filter (EKF), Unscented Kalman Filter (UKF), and Particle Filter (PF) [17, 18]. The particle filter has better performance for estimating parameters that are changing non-linearly at low Signal-to-Noise Ratio (SNR) $[17,18]$, so it will be used in the paper.

The paper is organized as follows: Section II presents the bistatic geometry of the passive radar system with the proposed method that depends on the particle filter. Section III explains the particle filter and its principles, taking into consideration the proposed method. Section IV illustrates the simulation of the mentioned system and discusses the simulation results. Section V concludes the paper.

\section{PASSIVE RADAR SYSTEM}

\section{A. Bistatic Geometry}

It consists of a DVB-T transmitter and one receiver, as shown in Fig. 1, taking into consideration that there is only 
one target, where $T_{x}$ is the non-cooperative transmitter, $R_{x}$ is the receiver with two receiving antennas, $T_{a}$ is the observed target, SC is the Surveillance Channel, RC is the Reference Channel, $R_{1}$ is the range between the transmitter $\left(T_{x}\right)$ and the target $\left(T_{a}\right), R_{2}$ is the effective range of the passive radar, $R_{b}$ is the bistatic range, $\mathrm{D}$ is the distance between the transmitter $\left(T_{x}\right)$ and the receiver $\left(R_{x}\right),\left(x_{a}, y_{a}, z_{a}\right)$ are the Cartesian coordinates of the target $\left(T_{a}\right)$, and $\beta$ is the bistatic angle.

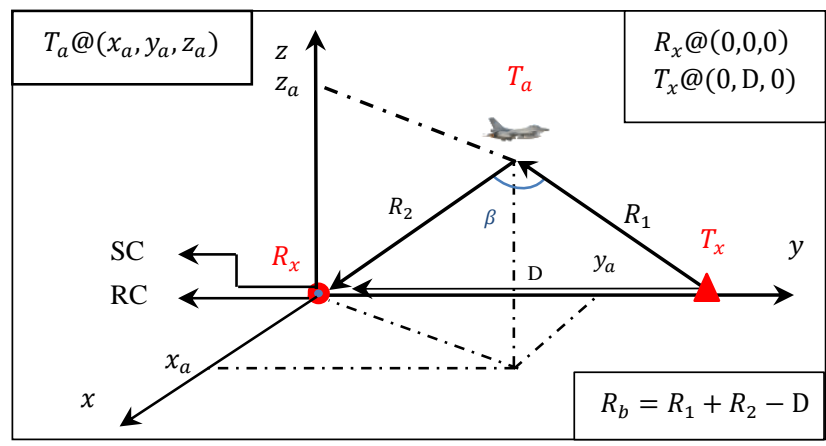

Fig. 1. Bistatic geometry for the passive radar system

\section{B. Proposed Method}

It depends on estimating and analyzing the Doppler frequency and time delay of the target's echo signal in the case of the described bistatic geometry. The target's echo signal is given in (1), taking into consideration that its parameters are: amplitude, phase, Doppler frequency, and time delay [17, 19, 20].

$$
y(t)=A(t) e^{j \varphi(t)} S(t-\tau)+n(t) ; t=0: T_{p}
$$

where $t$ is the observation time, $y$ is the echo signal of the observed target (or the observation signal), $A$ is the amplitude, $\varphi$ is the produced phase by the Doppler frequency $\left(f_{d}\right), S(t-$ $\tau)$ is the delayed reference signal with the time delay $(\tau), n$ is the Gaussian noise of the observation process, and $T_{p}$ is the duration of the processed data window.

The Doppler frequency and time delay are given in (2) and (3), respectively $[19,20]$, whereas the time delay is related to the target's coordinates, and the Doppler frequency is related to these coordinates and the Cartesian components of the target velocity.

$$
\begin{aligned}
f_{d_{t}}= & \frac{-1}{\lambda}\left[\frac{\left(x_{a_{t}}-x_{T}\right) v_{x}+\left(y_{a_{t}}-y_{T}\right) v_{y}+\left(z_{a_{t}}-z_{T}\right) v_{z}}{\sqrt{\left(x_{a_{t}}-x_{T}\right)^{2}+\left(y_{a_{t}}-y_{T}\right)^{2}+\left(z_{a_{t}}-z_{T}\right)^{2}}}\right. \\
& \left.+\frac{\left(x_{a_{t}}-x_{R}\right) v_{x}+\left(y_{a_{t}}-y_{R}\right) v_{y}+\left(z_{a_{t}}-z_{R}\right) v_{z}}{\sqrt{\left(x_{a_{t}}-x_{R}\right)^{2}+\left(y_{a_{t}}-y_{R}\right)^{2}+\left(z_{a_{t}}-z_{R}\right)^{2}}}\right] \\
\tau_{t} & =\tau_{1_{t}}+\tau_{2_{t}} \\
= & {\left[\sqrt{\left(x_{a_{t}}-x_{T}\right)^{2}+\left(y_{a_{t}}-y_{T}\right)^{2}+\left(z_{a_{t}}-z_{T}\right)^{2}}\right.} \\
& \left.+\sqrt{\left(x_{a_{t}}-x_{R}\right)^{2}+\left(y_{a_{t}}-y_{R}\right)^{2}+\left(z_{a_{t}}-z_{R}\right)^{2}}\right] / c
\end{aligned}
$$

where $\lambda$ is the carrier wavelength, $\left(x_{T}, y_{T}, z_{T}\right)$ $\&\left(x_{R}, y_{R}, z_{R}\right)$ are the transmitter and receiver coordinates, respectively, $\left(v_{x}, v_{y}, v_{z}\right)$ are the Cartesian components of the target velocity, $\tau_{1}$ is the time delay that corresponds to the range $\left(R_{1}\right), \tau_{2}$ is the time delay that corresponds to the range $\left(R_{2}\right)$, and $c$ is the speed of electromagnetic propagation.

Note: The time delay is the bistatic time delay, which is related to the ranges $\left(R_{1}, R_{2} \& D\right)$, as shown in Fig. 1. For simplicity, we will consider that this delay is only related to the ranges $\left(R_{1} \& R_{2}\right)$ because the range (D) is known, as given in (3).

According to (2) and (3), if the receiver can estimate the Doppler frequency and time delay, then the target's coordinates will be estimated by searching the coordinates that correspond to the estimated Doppler frequency and time delay. This is achieved by implementing the following two estimation stages: First, we estimate the Doppler frequency and time delay by the first particle filter. Second, we estimate these coordinates by the second particle filter depending on the estimated parameters from the first estimation stage.

For clarification, the role of the particle filter will be explained in the following section.

\section{PARTICLE FILTER}

\section{A. Introduction}

The Particle Filter is a method for implementing Recursive Bayesian Filter by Monte Carlo Sampling, whereas it depends on propagating in a non-linear way, of a set of weighted particles in a range of a studied state. The estimation results can be computed by processing particles' weights and states with helping from system observations. For better performance, the particles should be re-propagated (resampled) by using the resampling step [16], [20]-[23].

For each weighted particle, two equations should be processed for computing the estimation results. These two equations are the state equation and measurement equation, which are given in (4) and (5), respectively [16]-[24], where $t$ is the current measurement time, $(t-1)$ is the previous measurement time, $x$ is the state vector $\left(x \in \mathbb{R}^{n_{x}}\right), f$ is a nonlinear function and it is a known function, $v$ is the state noise vector that has a Gaussian distribution $\left(v \in \mathbb{R}^{n_{v}}\right)$; $v \sim \mathcal{N}\left(0, \sigma_{v}^{2}\right), Z$ is the measurement signal, and $h$ is a nonlinear function and it is a known function. The symbol $\left(\mathcal{N}\left(m, \sigma^{2}\right)\right)$ denotes the Gaussian density function with the mean $(m)$ and variance $\left(\sigma^{2}\right)$.

$$
\begin{aligned}
& x_{t}=f\left(x_{t-1}\right)+v_{t} \\
& Z_{t}=h\left(x_{t}\right)
\end{aligned}
$$

There are different types of the particle filter, such as the Maximum Likelihood Particle Filter (MLPF), Minimum Variance Particle Filter (MVPF), and Dirac Particle Filter (DPF). The type (MLPF) has less complexity with higher 
estimation accuracy compared to other types [17, 18]. Therefore, we will estimate the target's coordinates depending on the type (MLPF).

The Maximum Likelihood Particle Filter depends on the Likelihood function and Extended Kalman Filter for computing estimation results. It is achieved by implementing the following steps in each observation time, taking into consideration that the initial propagated particles have random states and equal weights; $\left\{w_{t=0}^{i}=1 / N_{s}, i=1: N_{s}\right\}$, where $w_{t=0}^{i}$ is the initial weight of the particle $(i), N_{s}$ is the number of particles, and $i$ is the index of these particles [16, 17], [21][24], (See the red particles in Fig. 2). The mentioned steps are as follows:

1) Approximating the Likelihood function $\left(p\left(y_{t} / x_{t}^{i}\right)\right)$, and then Updating the particles' weights based on the following equation, (See the brown curve and the blue particles in Fig. 2).

$$
\begin{aligned}
w_{t}^{i} & =w_{(t-1)}^{i} * p\left(y_{t} \mid x_{t}^{i}\right)=w_{(t-1)}^{i} * \mathcal{N}\left(y_{t}-h\left(x_{t}^{i}\right), R_{t}\right) \\
& =w_{(t-1)}^{i} * \mathcal{N}\left(y_{t}-Z_{t}^{i}, R_{t}\right)
\end{aligned}
$$

where $\left(w_{t}^{i}, w_{(t-1)}^{i}\right)$ are the current and previous weight for the particle $(i)$, respectively, $p$ is the probability density function (PDF), and $R$ is the covariance matrix [21, 24].

2) Normalizing the updated weights by the following equation.

$$
w_{t}^{i}=w_{t}^{i} / \sum_{i=1}^{N_{s}} w_{t}^{i}
$$

3) The estimated values are calculated by the following equation:

$$
\hat{x}_{t}=\sum_{i=1}^{N_{s}}\left(w_{t}^{i} * x_{t}^{i}\right)
$$

4) For better estimation, the particles that have higher weights should be selected for re-propagating other weighted particles in another range of the studied state. This is achieved by the resampling step, whereas the weights of the resampled particles are: $\left\{w_{t}^{i}=\left(1 / N_{S}\right) ; i=1: N_{S}\right\}$. See the blue and green particles in Fig. 2, [21]-[24].

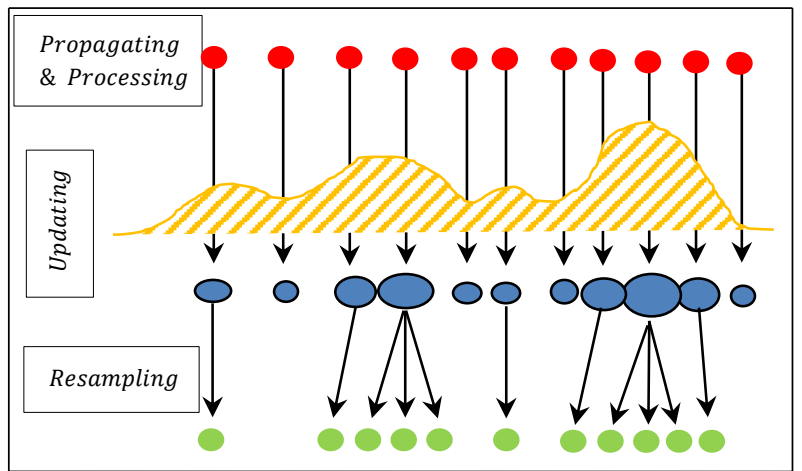

Fig. 2. Representation of steps of the particle filter
We have mentioned that the target's coordinates can be estimated by using two-particle filters in two consecutive estimation stages. For clarification, the state and measurement equations of these two filters will be illustrated in the following subsection, taking into consideration the time between the observations of the studied system.

\section{B. Equations of Two Particle Filters}

\section{B.1 Equations of the First Particle Filter}

1) State equation: It is related to the following parameters of the target's echo signal (amplitude, phase, Doppler frequency, and time delay). It is described in (9), [19, 20], where $x_{1}$ is the state vector of the first particle filter, $i_{1}$ is the index of the filter's particles; $\left(i_{1}=1: N_{s_{1}}\right), N_{s_{1}}$ is the number of the filter's particles, $\left(v^{A}, v^{\varphi}, v^{f_{d}}, v^{\tau}\right)$ are the Gaussian noises, and $f_{0}$ is the carrier frequency.

$$
x_{1_{t}}^{i_{1}}=\left[\begin{array}{c}
A_{t}^{i_{1}} \\
\varphi_{t}^{i_{1}} \\
f_{d_{t}}^{i_{1}} \\
\tau_{t}^{i_{1}}
\end{array}\right]=\left[\begin{array}{c}
A_{t-1}^{i_{1}} \\
\varphi_{t-1}^{i_{1}}+2 \pi f_{d_{t-1}}^{i_{1}} T_{p} \\
f_{d_{t-1}}^{i_{1}} \\
\tau_{t-1}^{i_{1}}-\frac{f_{d_{t-1}}^{i_{1}}}{f_{0}} T_{p}
\end{array}\right]+\left[\begin{array}{c}
v_{t}^{A} \\
v_{t}^{\varphi} \\
v_{t}^{f_{d}} \\
v_{t}^{\tau}
\end{array}\right]
$$

2) Measurement equation: It is given in (10), [19, 20].

$$
\begin{array}{r}
Z_{t}^{i_{1}}=A_{t}^{i_{1}} e^{j \varphi_{t}^{i_{1}}} S\left(t-\tau_{t}^{i_{1}}\right) \\
\left(t=0: T_{p}\right)
\end{array}
$$

\section{B.2 Equations of the Second Particle Filter}

1) State equation: It is related to the parameters of the target movement in Cartesian space. It is described in (11), [19], where $x_{2}$ is the state vector of the second particle filter, $i_{2}$ is the index of the filter's particles; $\left(i_{2}=1: N_{S_{2}}\right), N_{S_{2}}$ is the number of the filter's particles, $\left(\varepsilon x_{a}, \varepsilon y_{a}, \varepsilon z_{a}\right)$ are the Gaussian noises that are related to the state vector of the position, and $\left(\varepsilon v_{x}, \varepsilon v_{y}, \varepsilon v_{z}\right)$ are the Gaussian noises that are related to the state vector of the velocity components.

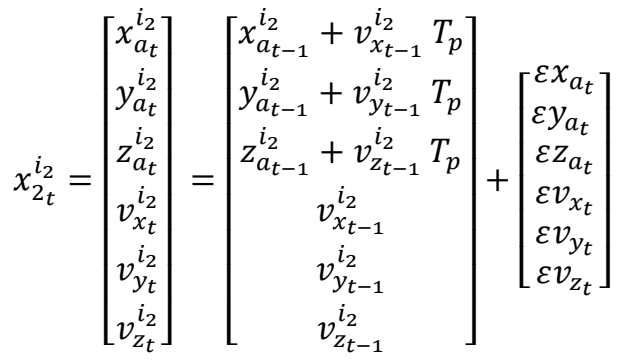

2) Measurement equation: It is given in (12), [19, 20], where $X_{a}$ is the target's position vector, $X_{T}$ is the transmitter's position vector, $X_{R}$ is the receiver's position vector, $V$ is the target's velocity vector, and \|\| is the norm of a vector. 


$$
Z_{t}^{i_{2}}=\left[\begin{array}{c}
f_{d_{t}}^{i_{2}} \\
\tau_{t}^{i_{2}}
\end{array}\right]=\left[\begin{array}{c}
\frac{-1}{\lambda}\left[\frac{\left(X_{a_{t}}^{i_{2}}-X_{T}\right) V_{t}^{i_{2}}}{\left\|X_{a_{t}}^{i_{2}}-X_{T}\right\|}+\frac{\left(X_{a_{t}}^{i_{2}}-X_{R}\right) V_{t}^{i_{2}}}{\left\|X_{a_{t}}^{i_{2}}-X_{R}\right\|}\right. \\
\frac{\left\|X_{a_{t}}^{i_{2}}-X_{T}\right\|+\left\|X_{a_{t}}^{i_{2}}-X_{R}\right\|}{c}
\end{array}\right]
$$

Note: The observation signal of this (PF) depends on the estimated Doppler frequency and time delay from the first PF.

By focusing on (3) and taking into consideration the proposed method, we notice that the summation in this equation affects the estimation of the target's coordinates with ambiguity in the estimation. This ambiguity is related to infinite probabilities giving the same result of the summation. Therefore, the estimated coordinates will be estimated with ambiguity. To complete this estimation correctly without ambiguity, we will depend on the estimated Cartesian components of the target velocity. But this approach cannot be completed without initial values of the target's coordinates, whereas these values can be taken from results of other researches or by using a third particle filter. For clarification, we will consider that the estimated coordinates "with ambiguity" are the primary estimated coordinates, and the other estimated coordinates are the corrected estimated coordinates.

Figure-3 shows the flow chart of the proposed method, where the symbol $\left(^{\wedge}\right)$ refers to an estimated parameter, $\left[\begin{array}{lll}x_{a_{0}} & y_{a_{0}} & z_{a_{0}}\end{array}\right]^{T}$ indicates the initial coordinates of the target, $T$ is the transposition, $\Delta t$ is the time difference between two consecutive observations, and $\hat{v}_{e}$ is the estimated velocity.

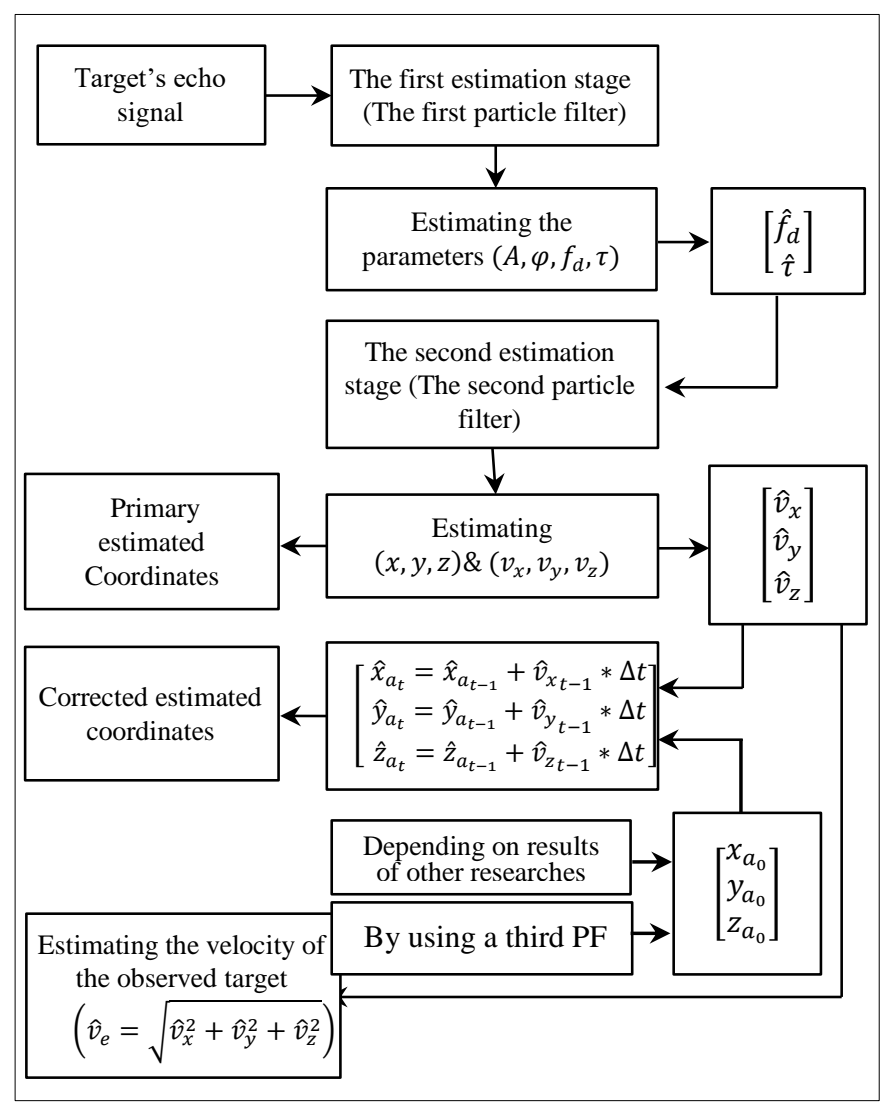

Fig. 3. Flow chart of the proposed method

\section{Simulation AND RESUlts}

\section{A. Simulation}

MATLAB software is used for simulating the passive radar system that consists of a DVB-T transmitter [25], the radar receiver, and the Gaussian Noise channel with one observed target. To complete the description of this simulation, we will add the technical characteristics of the components of the mentioned system, as listed in Table I, where ERP refers to (Effective Radiated Power), and OFDM refers to (Orthogonal Frequency Division Multiplexing).

TABLE I

TeChNiCAl CharaCteristics Of TRANSMItTER, RECEIVER, AND TARGET

\begin{tabular}{|c|c|c|c|c|}
\hline \multirow{4}{*}{ 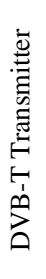 } & ERP & $50(\mathrm{KW})$ & Cyclic Prefix & $1 / 4$ \\
\hline & $\begin{array}{l}\text { Carrier } \\
\text { Frequency }\end{array}$ & $474(\mathrm{MHz})$ & $\begin{array}{l}\text { Cartesian } \\
\text { Coordinates }\end{array}$ & $(0, D, 0)$ \\
\hline & Bandwidth & $8(\mathrm{MHz})$ & $\mathrm{D}$ & $5(\mathrm{Km})$ \\
\hline & $\begin{array}{l}\text { Transmission } \\
\text { Mode }\end{array}$ & $\begin{array}{l}8 \mathrm{~K} \text { mode/ } \\
64 \mathrm{QAM}\end{array}$ & Losses & $1(\mathrm{~dB})$ \\
\hline \multirow{4}{*}{ 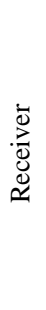 } & $\begin{array}{l}\text { Gain of } \\
\text { Surveillance } \\
\text { Antenna }\end{array}$ & $22(\mathrm{~dB})$ & Losses & $1(\mathrm{~dB})$ \\
\hline & $\begin{array}{l}\text { Gain of } \\
\text { Reference } \\
\text { Antenna } \\
\end{array}$ & $2.5(\mathrm{~dB})$ & $\Delta t$ & 0.1499 (s) \\
\hline & $\begin{array}{l}\text { Cartesian } \\
\text { Coordinates }\end{array}$ & $(0,0,0)$ & $T_{p}$ & $2.2(\mathrm{~ms})$ \\
\hline & Noise Figure & $2(\mathrm{~dB})$ & & \\
\hline 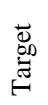 & $\begin{array}{l}\text { Monostatic } \\
\text { RCS }\end{array}$ & $6\left(\mathrm{~m}^{2}\right)$ & $\begin{array}{l}\text { Initial } \\
\text { Coordinates }\end{array}$ & $\begin{array}{l}(8,8,3) \\
(\mathrm{Km})\end{array}$ \\
\hline
\end{tabular}

We consider that the observed target moves according to the trajectory shown in Fig. 4, and its velocity changes according to Fig. 5. Therefore, the (SNR) of the target's echo signal changes according to the range $[9.8 \rightarrow 17.1](d B)$, and the mentioned target is detected with a false alarm probability of: $\left(10^{-4}\right)$.

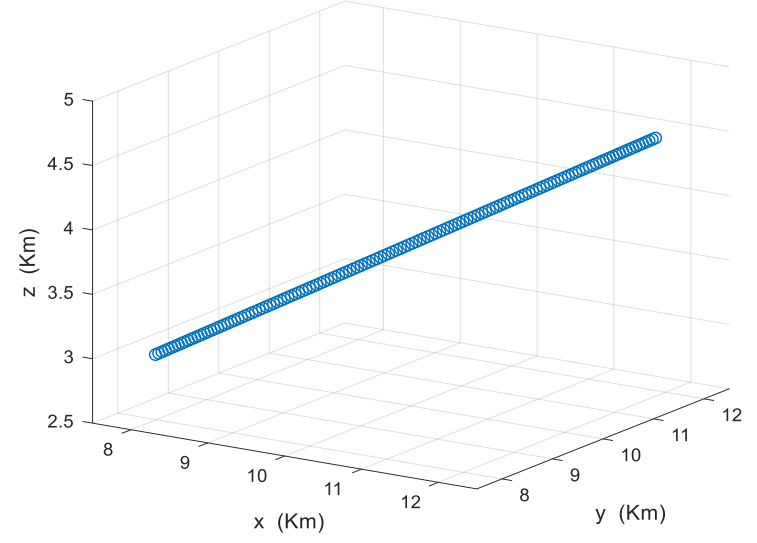

Fig. 4. Trajectory of the observed target. 


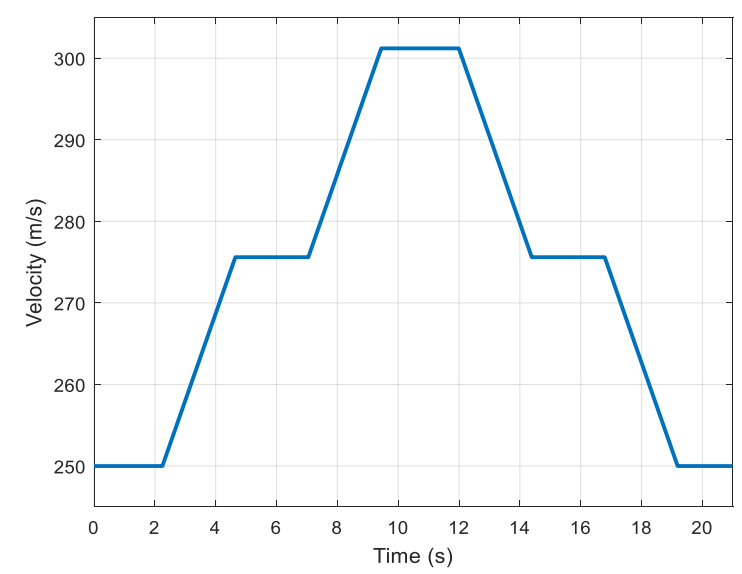

Fig. 5. Velocity of the observed target as a function of time

This simulation has been achieved with the following considerations:

1) The target's echo signal is detected by correlating the reference signal with the surveillance signal. This process is achieved by applying the Maximum Likelihood method to the output of a bank of matched filters, which are tuned to different Doppler frequencies [1, 10, 19].

2) The range of the Signal-to-Interference ratio (SIR) is $[-70.8 \rightarrow-63.2](d B)$, whereas this parameter is very important for detecting the target's echo signal in the surveillance channel [7].

3) Estimation accuracy is related to the standard deviation of estimation errors. It is given in $(13),[16,18]$, where $\sigma_{E A}$ is the Estimation Accuracy of the studied parameter, $M$ is the number of observations, $d$ is the estimation error that has the equation: $\left(d_{i}={\text { true } \text { value }_{i}-\text { estimated value }}_{i}\right)$, and $\mu$ is the mean of estimation errors.

$$
\sigma_{E A}=\sqrt{\frac{1}{M-1} \sum_{i=1}^{M}\left(d_{i}-\mu\right)^{2}}
$$

4) The initial coordinates of the observed target are taken from the method of [15], whereas authors of this reference studied estimating the target's coordinates by analyzing the bistatic geometry of passive radar with a single noncooperative transmitter and a single receiver.

5) The movement of targets at high velocities imposes a noise on the state vector of a studied system, whereas it is uncorrelated with the state noise vector. This noise is called Dynamic Noise, and it is Gaussian noise with a variance and zero mean $[17,18]$. We will list its Gaussian distribution in the case of our parameters as follows, where $(D N)$ is the Dynamic Noise, and (nor) refers to "normalized".

- $\quad D N^{A} \sim \mathcal{N}\left(0,0.01^{2}\right.$ (nor) $)$

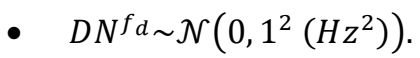

- $\quad D N^{\tau} \sim \mathcal{N}\left(0,0.0035^{2}\right.$ (nor $\left.)\right)$.

- $\quad D N^{\text {Position }} \sim \mathcal{N}\left(0,1^{2}\left(m^{2}\right)\right)$.

- $\quad D N^{\text {Velocity }} \sim \mathcal{N}\left(0,0.1^{2}\left(\frac{m}{s}\right)^{2}\right)$.

6) The parameters of those two consecutive particle filters are listed in Table II, where $(\sigma)$ is the standard deviation, [17][19].

TABLE II

Parameters Of Two Consecutive Particle Filters

\begin{tabular}{|c|c|c|c|c|}
\hline$\stackrel{\mathscr{e}}{\stackrel{C}{C}}$ & $N_{s_{1}}$ & 27 & $\sigma_{w^{A}}($ normalized) & 0.001 \\
\hline 窝 & $\sigma_{w^{f_{d}}}(\mathrm{~Hz})$ & 0.1 & $\sigma_{w^{\tau}}($ normalized $)$ & 0.001 \\
\hline \multirow{5}{*}{ 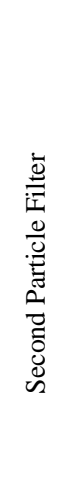 } & $N_{s_{2}}$ & 300 & $\sigma_{\varepsilon v_{x}}(\mathrm{~m} / \mathrm{s})$ & 0.05 \\
\hline & $\sigma_{\varepsilon x_{a}}(m)$ & 0.5 & $\sigma_{\varepsilon v_{y}}(\mathrm{~m} / \mathrm{s})$ & 0.05 \\
\hline & $\sigma_{\varepsilon y_{a}}(m)$ & 0.5 & $\sigma_{\varepsilon v_{z}}(\mathrm{~m} / \mathrm{s})$ & 0.05 \\
\hline & $\sigma_{\varepsilon z_{a}}(m)$ & 0.5 & & \\
\hline & $\begin{array}{l}\text { Standard deviations } \\
\text { of initial coordinates } \\
(m)\end{array}$ & {$\left[\begin{array}{l}86 \\
95 \\
17\end{array}\right]$} & $\begin{array}{l}\text { Standard deviations } \\
\text { of initial velocity } \\
\text { components }(\mathrm{m} / \mathrm{s})\end{array}$ & {$\left[\begin{array}{c}12 \\
12 \\
6\end{array}\right]$} \\
\hline
\end{tabular}

\section{B. Results}

After performing the simulation of the passive radar system, we obtained the estimated parameters (amplitude, Doppler frequency, and time delay) resulting from the first estimation stage, whereas the estimation accuracies were as follows: $\sigma_{A}=0.012$ (normalized), $\sigma_{f_{d}}=1.24(\mathrm{~Hz})$ and $\sigma_{\tau}=$ 0.0039 (normalized). See figures $(6,7$, and 8$)$ that show the results of the first estimation stage.

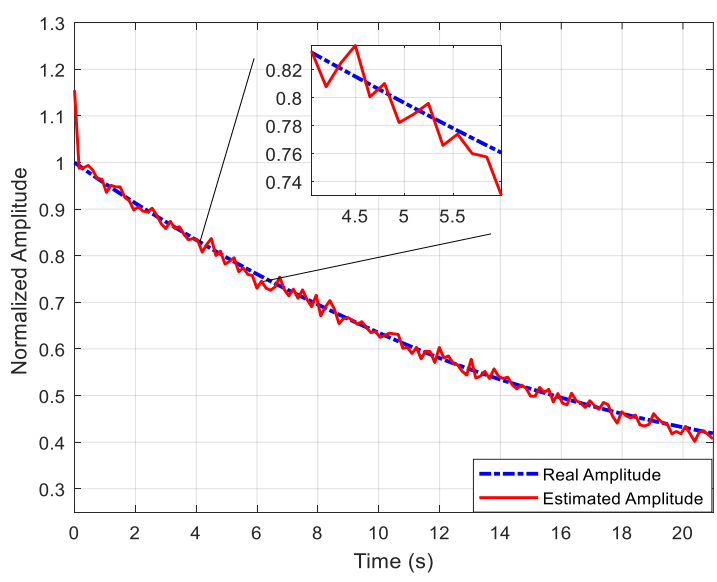

Fig. 6. Real and estimated amplitude as a function of time 


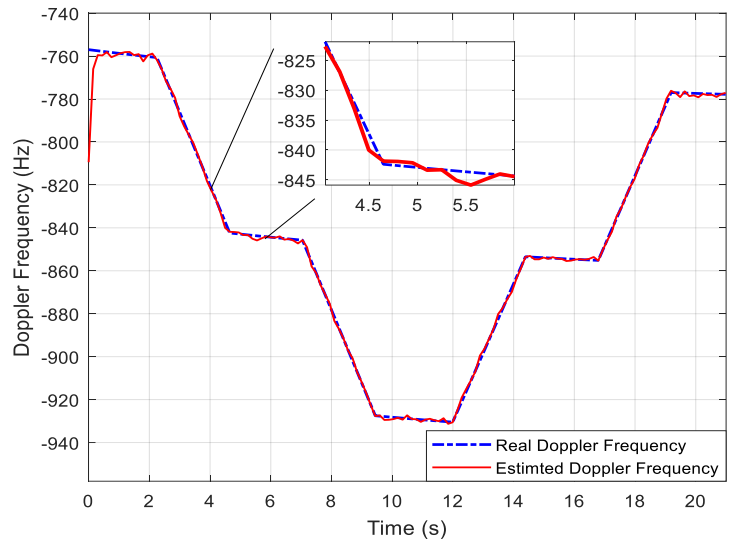

Fig. 7. Real and estimated Doppler frequency as a function of time

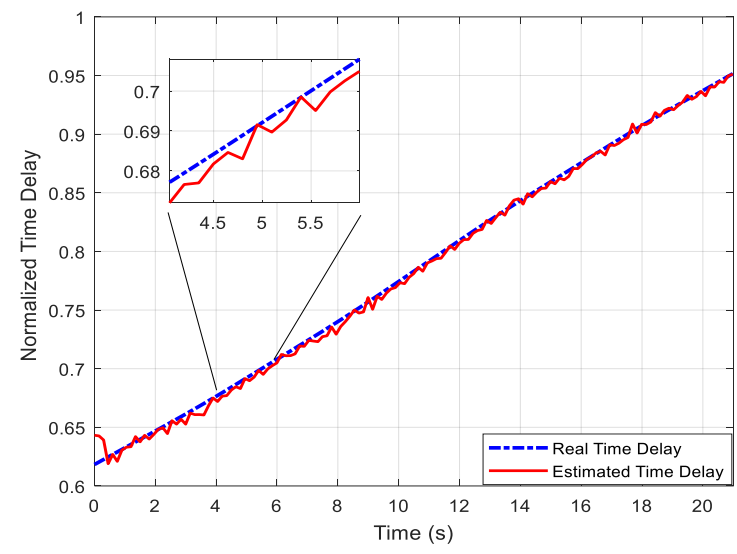

Fig. 8. Real and estimated time delay as a function of time

After performing the second estimation stage based on the results of the first estimation stage, we can obtain the primary coordinates, the corrected coordinates, and the Cartesian components of the target velocity. To verify the efficiency of the proposed method, we will show the estimated parameters as follows: First, the primary and corrected coordinates, as shown in Fig. 9 and Fig. 10, respectively. Second, the velocity of the target, which is estimated by calculating the resultant of the estimated Cartesian components of the target velocity, as shown in Fig. 11. The estimation accuracy of the target velocity is $\left(\sigma_{\text {velocity }}=0.74(\mathrm{~m} / \mathrm{s})\right)$.
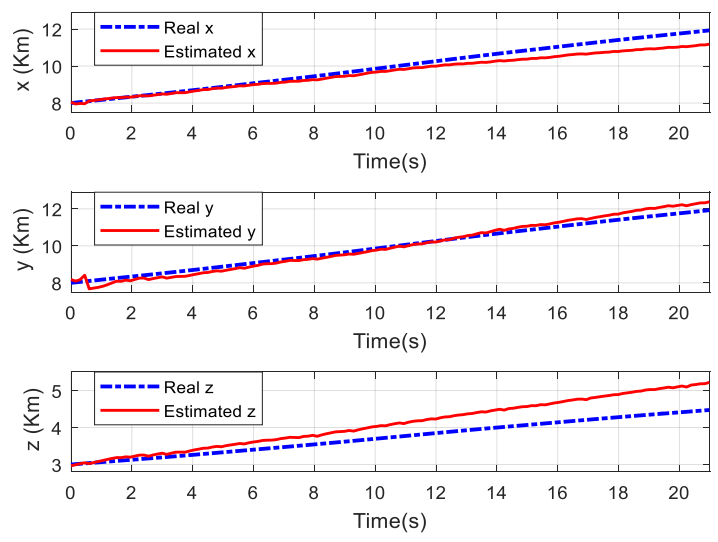

Fig. 9. "Real" and "primary estimated" coordinates as a function of time
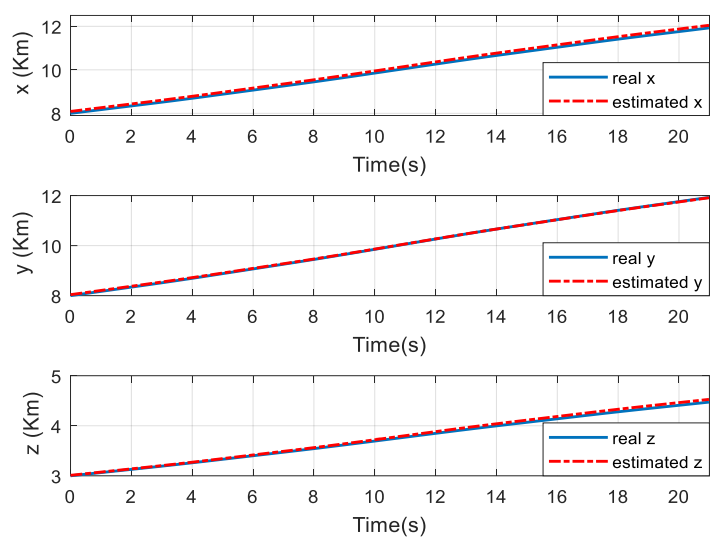

Fig. 10. "Real" and "corrected estimated" coordinates as a function of time

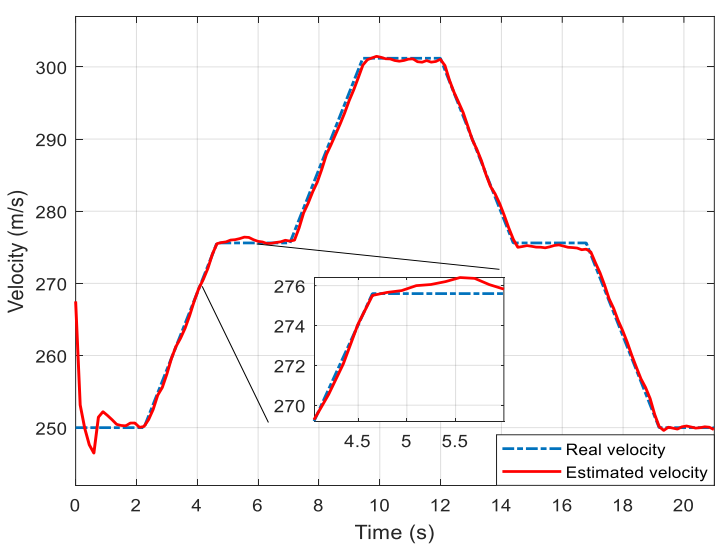

Fig. 11. Real and estimated velocity of the target as a function of time

Note: We have mentioned that the primary estimated coordinates are not the right ones, meanwhile they lead to the same time delay that corresponds to the corrected estimated coordinates, as shown in Fig. 12.

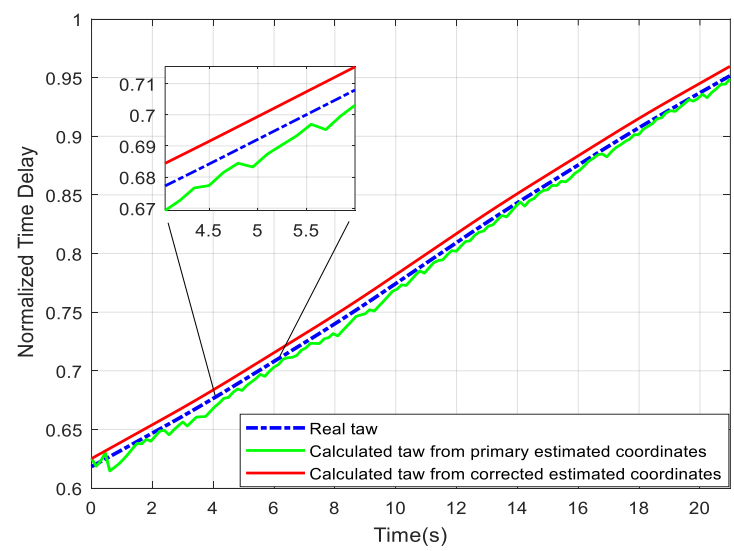

Fig. 12. Real and calculated time delay as a function of time

\section{Discussing the Simulation Results}

By focusing on the simulation results, we notice the following points:

- The estimation accuracies of the corrected estimated coordinates are related to the standard deviations of 
the initial coordinates.

- The target's coordinates have been estimated for the observed target that moves along the specific trajectory, which was a straight trajectory. In the case of a maneuvering target, having a "zigzag" trajectory, for example, the proposed method fails, and the target's coordinates cannot be estimated correctly. To be able to estimate them correctly in that case of the "zigzag" trajectory, we need to determine/estimate the direction of the target according to the axes of Cartesian space.

- Processing the passive radar with bistatic geometry "One Non-cooperative Transmitter / One Receiver" overrides the disadvantages of Multistatic Passive Radars [15].

- The effectiveness of this method has been compared with that of the method of [15]. Comparison results are illustrated in the following table.

TABLE III

COMPARISON WITH THE METHOD OF [15]

\begin{tabular}{|c|c|c|}
\hline & Method of this paper & Method of [15] \\
\hline Bistatic geometry & $\begin{array}{l}\text { One Pair } \\
\left(T_{x}-R_{x}\right)\end{array}$ & $\begin{array}{l}\text { One Pair } \\
\left(T_{x}-R_{x}\right)\end{array}$ \\
\hline Complexity & More complexity & Less complexity \\
\hline $\begin{array}{l}\text { Estimation accuracies } \\
\text { of target's coordinates }\end{array}$ & $\begin{array}{l}\text { Errors are related to } \\
\text { the standard deviations } \\
\text { of the initial } \\
\text { coordinates }\end{array}$ & $\begin{array}{l}\text { Errors are related to } \\
\text { the method of } \\
\text { processing }\end{array}$ \\
\hline $\begin{array}{l}\text { Estimation of target's } \\
\text { coordinates without the } \\
\text { need for initial } \\
\text { coordinates }\end{array}$ & $\begin{array}{c}\text { Not effective } \\
\text { (These initial } \\
\text { coordinates can be } \\
\text { taken from results of } \\
\text { other researches or by } \\
\text { using a third particle } \\
\text { filter) }\end{array}$ & Effective \\
\hline $\begin{array}{l}\text { Estimation of Doppler } \\
\text { frequency and velocity }\end{array}$ & More effective & Less effective \\
\hline $\begin{array}{l}\text { Estimation of target's } \\
\text { coordinates in the case } \\
\text { of a slight zigzag } \\
\text { trajectory }\end{array}$ & Effective & Effective \\
\hline $\begin{array}{l}\text { Estimation of target's } \\
\text { coordinates in the case } \\
\text { of a strong zigzag } \\
\text { trajectory }\end{array}$ & Less effective & More effective \\
\hline
\end{tabular}

- Integrating the method of this paper with the method of [15] can improve the performance of the proposed passive radar for tracking targets in many spaces, such as "Cartesian space", "Spherical space", "Doppler FrequencyTime delay", and "Velocity-effective range".

\section{CONCLUSION}

In this paper, a new method has been proposed for localizing a target in Cartesian Space by passive radar that has a single bistatic geometry (one DVB-T transmitter and one receiver). This method depends on estimating and analyzing the Doppler frequency and time delay of the target's echo signal, by using two consecutive particle filters. By performing the simulation of the proposed passive radar system, we have achieved localization of a target in Cartesian space by estimating its Cartesian coordinates. The effectiveness of the proposed method has been illustrated by comparing the simulation results with other researches.

\section{REFERENCES}

[1] H. Kuschel, D. Cristallini, and K. E. Olsen: Tutorial: Passive Radar Tutorial, IEEE Aerospace and Electronic Systems Magazine, Vol.34, No.2, pp. 2-19, 2019, DOI: 10.1109/MAES.2018.160146.

[2] X. Zhang, J. Yi, X. Wan, and Y. Liu: Reference Signal Reconstruction Under Oversampling for DTMB-Based Passive Radar, IEEE Access 8, pp. 74024-74038, 2020. DOI: 10.1109/ACCESS.2020.2986589.

[3] W. Cao, X. Li, W. Hu, J. Lei, and W. Zhang: OFDM reference signal reconstruction exploiting subcarrier-grouping-based multi-level LloydMax algorithm in passive radar systems, IET Radar, Sonar \& Navigation, Vol.11, Iss.5, pp. 873-879, 2017, DOI: 10.1049/ietrsn.2016.0340.

[4] M. Płotka, M. Malanowski, P. Samczyński, K. Kulpa, and K Abratkiewicz: Passive Bistatic Radar Based on VHF DVB-T Signal, In 2020 IEEE International Radar Conference (RADAR), 2020, pp. 596600, DOI: $10.1109 /$ radar42522.2020.9114859.

[5] H. D. Griffiths: PASSIVE BISTATIC RADAR AND WAVEFORM DIVERSITY, Defense Academy of the United Kingdom Shrivenham, United Kingdom, 2009

[6] B. Satar, G. Soysal, X. Jiang, M. Efe, and T. Kirubarajan: Robust Weighted $l_{1,2}$ Norm Filtering in Passive Radar Systems, Sensors, Vol.20, No.11, 2020, DOI: 10.3390/s20113270.

[7] G. E. Lange: Performance Prediction and Improvement of a Bistatic Passive Coherent Location Radar, M.S. thesis, Department of Electrical Engineering, University of Cape Town, 2009.

[8] O. Mahfoudia, F. Horlin, and X. Neyt: Pilot-based detection for DVB-T passive coherent location radars, IET Radar, Sonar \& Navigation, Vol.14, Iss.6, pp. 845-851, 2020, DOI: 10.1049/ietrsn.2019.0268.

[9] Y. Zhou, W. Xia, J. Zhou, L. Huang and M. Huang: Coherent Integration Algorithm for Weak Maneuvering Target Detection in Passive Radar Using Digital TV Signals, In International Conference on Machine Learning and Intelligent Communications, Springer, Cham, 2017, pp. 215-224.

[10] L. Zheng and X. Wang: Super-Resolution Delay-Doppler Estimation for OFDM Passive Radar, IEEE Transactions on Signal Processing, Vol.65, No.9, pp. 2197-2210, 2017, DOI:. 10.1109/TSP.2017.2659650.

[11] A. Aubry, V. Carotenuto, A. D. Maio, and L. Pallotta: Localization in 2D PBR With Multiple Transmitters of Opportunity: A Constrained Least Squares Approach, IEEE Transactions on Signal Processing, pp. 634-646, 2020, DOI: 10.1109/TSP.2020.2964235.

[12] M. Malanowski: Algorithm for Target Tracking Using Passive Radar, International Journal of Electronics and Telecommunications, Vol.58, No.4, pp. 345-350, 2012, DOI: 10.2478/v10177-012-0047-x.

[13] M. Malanowski, and K. Kulpa: Two Methods for Target Localization in Multistatic Passive Radar, IEEE transactions on Aerospace and Electronic Systems, Vol.48, No.1, pp. 572-580, 2012, DOI: 10.1109/taes.2012.6129656.

[14] J. Wang, Z. Qin, F. Gao, and S. Wei: An Approximate Maximum Likelihood Algorithm for Target Localization in Multistatic Passive Radar, Chinese Journal of Electronics, Vol.28, Iss.1, pp.195-201, 2019, DOI: $10.1049 /$ cje.2018.02.018. 
[15] A. Kazem, A. Malki, and A. M. Almanofi: Target Coordinates Estimation by Passive Radar with a Single non-Cooperative Transmitter and a Single Receiver, Journal of Communications Software and Systems, Vol.16, No.2, pp. 156-162, 2020, DOI: 10.24138/jcomss.v16i2.984.

[16] A. M. Almanofi, A. Malki, and A. Kazem: Doppler Frequency Estimation for a Maneuvering Target Being Tracked by Passive Radar Using Particle Filter, Journal of Communications Software and Systems, Vol.16, No.4, pp. 279-284, DOI: 10.24138/jcomss.v16i4.1097.

[17] A. Kazem: Generalized Deterministic Particles in non-Linear Filtering. Defense and Communication Applications, Ph.D. dissertation, LAAS, France, 2008, (In French).

[18] A. Ziadi: Deterministic Gaussian Particles in Non-Linear Maximum Likelihood: Application of optimal Filtering in Radar and GPS Signals, Ph.D. dissertation, LAAS, France, 2007, (In French).

[19] K. Jishy: Tracking Maneuvering Targets in case of Passive Radar with Gaussian Particle filter, Ph.D. dissertation, National Institute of Telecommunications, Pierre and Marie Curie University, Paris, France, 2012, (In French).

[20] K. Jishy and F. Lehmann: A Bayesian track-before-detect procedure for passive radars, EURASIP Journal on Advances in Signal Processing, No.1, 2013, DOI: 10.1186/1687-6180-2013-45.

[21] M. S. Arulampalam, S. Maskell, N. Gordon, and T. Clapp: A Tutorial on Particle Filters for Online Nonlinear/Non-Gaussian Bayesian Tracking, IEEE Transactions on signal processing, Vol.50, No.2, pp. 174-188, 2002, DOI: $10.1109 / 78.978374$.

[22] J. Elfring, E. Torta, and R. v. Molengraft: Particle Filters: A Hands-On Tutorial, Sensors, vol. 21, no. 2, 2021, DOI: 10.3390/s21020438.

[23] M. Speekenbrink: A tutorial on particle filters, Journal of Mathematical Psychology, vol. 73, pp. 140-152, 2016, DOI: 10.1016/j.jmp.2016. 05.006.

[24] F. Gustafsson: Particle Filter Theory and Practice with Positioning Applications, IEEE Aerospace and Electronic Systems Magazine, Vol.25, No.7, pp. 53-82, 2010, DOI: 10.1109/maes.2010.5546308.

[25] ETSI EN 300744 v1.6.1 (2009-01): Digital Video Broadcasting $(D V B) ; \quad$ Framing structure, channel coding and modulation for digital terrestrial television, European Standard (Telecommunications series), 2009. [Online]. Available: https://www.etsi.org/deliver/etsi_en/300700_300799/300744/01.06.01_6 0/en 300744v010601p.pdf.

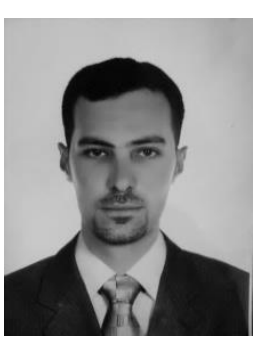

A. M. Almanofi was born in Damascus, Syrian Arab Republic in 1987. Received the Communication and Electronics engineering degree in 2011 from Damascus University. He received his Master degree in Communication from the Higher Institute for Applied Sciences and Technology (HIAST) in 2015 $\mathrm{He}$ is currently pursuing the Ph.D. degree in Passive Radars at (HIAST). His research interests are in localizing targets in Cartesian space and estimating parameters of maneuvering targets by Particle Filter.

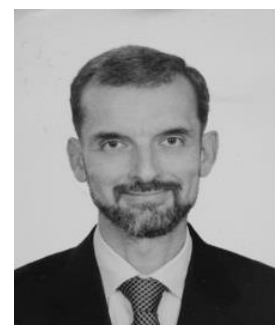

A. Malki was born in Damascus, Syrian Arab Republic in 1956. He received his Electrical Engineering degree (Honor) in 1979, from Damascus University. He received his DEA degree in Electronics in 1982, from the National Higher School of Aeronautics and Space, Toulouse, France. $\mathrm{He}$ received his Ph.D. degree in Electronics/ Microwave Circuit Design in 1985, from the National Higher School of Aeronautics and Space, Toulouse, France. During that period he worked with Thomson CSF, Space division at Toulouse, France. His main research interests include: design and development of a wide variety of microwave components and subsystems, such as filters, directional couplers, detectors, LNAs, medium and high power amplifiers, frequency synthesizers, RF and Microwave front ends. He is a senior professor at the Higher Institute for Applied Sciences and Technology (HIAST), Damascus, Syria.

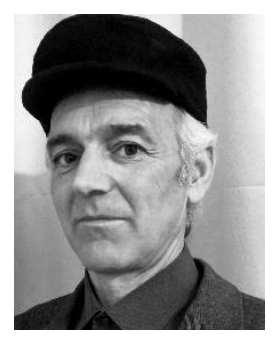

A. Kazem was born in Homs, Syrian Arab Republic in 1970. He received the Engineering and DES degrees in 1994, from the Higher Institute for Applied Sciences and Technology (HIAST), Damascus, Syria. He received his DEA degree in Automatics and Signal Processing in 2003, from the Laboratory for Analysis and Architecture of Systems (LAAS), Toulouse, France. In 2007, he received the Ph.D. degree in Random Signal Processing/Nonlinear Filtering from LAAS-CNRS. He worked as a research engineer with HIAST, Alcatel Alena Space-France, and DSI (Distribution Services Industrials), Toulouse, France. His research interests include statistical signal processing and its applications in radar, sonar, wireless communication systems, and identification systems. Since 2010, he has been a researcher in the Department of Electronic Systems, HIAST, Damascus-Syria. 